\title{
Antimicrobial susceptibility of uropathogens and prescribing patterns in hospital- and community-acquired urinary tract infections in a tertiary care hospital
}

\author{
Achukutty Tomy ${ }^{1}$, Aravind Hareendran ${ }^{1}$, Emil David ${ }^{1}$, Ravina Ravi ${ }^{1}$, Lakshmi Ramachandran ${ }^{1 *}$, Appu Thomas ${ }^{2}$ \\ ${ }^{1}$ Department of Pharmacy Practice, Amrita School of Pharmacy, AIMS Health Science Campus, Kochi, India. \\ ${ }^{2}$ Department of Urology, Amrita Institute of Medical Sciences and Research Centre, AIMS Health Science Campus, Kochi, India.
}

\begin{tabular}{|c|c|}
\hline ARTICLE INFO & ABSTRACT \\
\hline $\begin{array}{l}\text { Received on: } 25 / 05 / 2020 \\
\text { Accepted on: } 04 / 10 / 2020 \\
\text { Available online: } 05 / 11 / 2020\end{array}$ & $\begin{array}{l}\text { Periodic surveillances on the prevalence and antimicrobial susceptibility of uropathogens are necessary as they } \\
\text { vary with time and geography. This prospective study for } 8 \text { months included } 331 \text { and } 335 \text { patients with hospital- } \\
\text { acquired and community-acquired urinary tract infections (HA-UTI and CA-UTI), respectively. The prevalence of } \\
\text { Gram-negative bacilli was higher in both groups, with Escherichia coli being the most prevalent uropathogen in both }\end{array}$ \\
\hline $\begin{array}{l}\text { Key words: } \\
\text { Urinary tract infections, } \\
\text { antimicrobial susceptibility, } \\
\text { multiple drug resistance, } \\
\text { multiple antibiotic resistance } \\
\text { index, antimicrobial } \\
\text { stewardship. }\end{array}$ & $\begin{array}{l}\text { groups. An alarming number of isolates with multiple drug resistance was observed in HA-UTI and CA-UTI ( } 47 \% \text { and } \\
46.2 \% \text {, respectively). The mean multiple antibiotic resistance index in both groups was }>0.2 \text {. Prescribing patterns and } \\
\text { percentage antimicrobial susceptibility antibiograms revealed high resistance to commonly prescribed antimicrobials } \\
\text { like } \beta \text {-lactams and fluoroquinolones. Retrospective data of the preceding } 2 \text { years revealed a statistically significant } \\
\text { reduction in antimicrobial sensitivity of E. coli and Klebsiella isolates to several antibiotics. Antibiotic resistance is a } \\
\text { major threat to patient safety in the hospital and the community. Strengthening of antimicrobial stewardship strategies } \\
\text { like continued antimicrobial susceptibility surveillance, framing empirical antimicrobial policies, and promoting } \\
\text { rational prescription of antimicrobials is the need of the hour. }\end{array}$ \\
\hline
\end{tabular}

\section{INTRODUCTION}

With the advent of the "golden era of antibiotics", human life expectancy significantly increased by cure of previously fatal infections, but the emergence of antibiotic resistance has become a global concern as it has large clinical and financial burden and needs urgent action (Kaur et al., 2016). Standard treatments become ineffective and infections persist due to antimicrobial resistance. In a global review on antimicrobial resistance, 700,000 annual deaths attributable to antibiotic-resistant infections were estimated and are likely to rise to about 10 million by 2050 , if action is not taken to combat this condition whose effect is especially felt in countries

\footnotetext{
${ }^{*}$ Corresponding Author

Lakshmi Ramachandran, Department of Pharmacy Practice, Amrita School of Pharmacy, AIMS Health Science Campus, Kochi, India. E-mail: lakshmir87@gmail.com
}

referred to as the BRIC (Brazil, Russia, India, China) and MINT (Mexico, Indonesia, Nigeria, Turkey) (Joseph et al., 2017). In a developing country, like India, a high burden of resistant infections is of concern due to the high rate of poverty which constrains the access to more effective but more expensive antimicrobial agents.

Urinary tract infections (UTIs) are common in clinical practice and are responsible for a large proportion of antibiotic consumption in the hospital and the community. Rational antibiotic treatment of UTI is essential to keep antibiotic consumption low, so as to reduce the emergence of resistance in the hospital and the community (Frimodt, 2002). Hospitalacquired UTI (HA-UTI) and community-acquired UTI (CAUTI) differ in etiology, antimicrobial resistance profile, and prognosis (Agaba et al., 2017; Aguilar-Duran et al., 2012). The prevalence and susceptibility of uropathogens vary with time, geography, and from one institution to another (Mukherjee et al., 2013). All institutions should therefore have continuous antimicrobial surveillance of uropathogens and periodic hospital 
antibiograms prepared, since it not only helps clinicians to select appropriate empirical policies, but also to monitor resistance trends within an institution, thereby optimizing therapy.

This study aims to assess the antimicrobial susceptibility of uropathogens and prescribing pattern of antimicrobials in HA-UTI and CA-UTI and to identify any statistically significant reduction in antimicrobial sensitivity of common uropathogens during the preceding 2 years. The study assessed the prevalence of uropathogens, risk factors, and multiple drug resistance (MDR), and also calculated the multiple antibiotic resistance index (MARI).

\section{METHODOLOGY}

\section{Study design, selection of patients, and clinical data acquisition}

A single-center retroprospective study was conducted in the outpatient clinics of the Department of General Medicine, Gynecology, Nephrology, and Urology for the assessment of CA-UTI, and the assessment of HA-UTI was conducted in all inpatients admitted to Amrita Institute of Medical Sciences and Research Center, a tertiary care teaching hospital in Kerala, India. This prospective study was conducted from September 2018 to April 2019. Retrospective data from September 2016 to August 2018 were collected during this period. HA-UTIs are those that are identified after 48 hours of admission to hospital and CA-UTIs are those contracted outside of a healthcare setting or an infection present on admission, prior to development of the symptoms (Agaba et al., 2017; Aguilar-Duran et al., 2012). The study population consisted of patients aged $\geq 18$ years whose urine culture reports were positive with significant bacteriuria [a colony count of $\geq 10^{5}$ colony forming units (CFU) of bacteria per cc of urine in non-catheterized patients and $\geq 10^{2} \mathrm{CFU}$ of bacteria per cc of urine in catheterized patients] and antibiotic susceptibility tests were carried out (Hooton, 1990; Hooton et al., 2010). Patients presenting more than once during the study period were considered only during first visit. Data were collected from the hospital's digital information system and direct review of inpatient medical records using predesigned forms. The study (IEC-AIMS-2018-PHARM-171) was approved by the Institutional Ethics Committee and written informed consent was obtained from all study participants.

\section{Prospective data}

The prospective study included 331 HA-UTI patients [62 from Intensive Care Units (ICUs) and 269 from wards] and 335 CA-UTI patients [general medicine (94), gynecology (39), nephrology (74), and urology (128)] and from them 370 and 351 isolates of uropathogens was obtained, respectively. Patient demographics, risk factors, and prevalence of uropathogens were assessed. Culture and sensitivity (C\&S) reports were analyzed to identify MDR isolates (non-susceptibility to at least one agent in three or more antimicrobial categories) and MARI was calculated (Magiorakos et al., 2012). MARI, a tool for health risk assessment, ascertains if isolates are from a region of high or low antibiotic usage. MARI value ranges from 0 to 1 , and values $>0.2$ indicates "high-risk" sources of antibiotic resistance, wherein antibiotics are frequently used or misused. This indicates that large proportions of bacterial isolates have developed resistance through exposure to several antibiotics (Köves et al., 2017). MARI is determined by dividing the number of antimicrobials to which a pathogen is resistant by the total number of antimicrobials to which it was exposed (Chacko et al., 2017; Mitchell et al., 2016). Percentage susceptibilities of isolated uropathogens to routinely tested antimicrobials were represented in cumulative hospital antibiograms and developed according to the Clinical Laboratory Standard Institute guidelines on Analysis and Presentation of Cumulative Antimicrobial Susceptibility Testing. Species with less than 10 isolates have not been included due to sample size limitations. Organisms with 10-30 isolates have been included but were interpreted with caution, as small numbers may bias group susceptibilities. Intermediate sensitivity has not been included. Antimicrobial prescriptions before and after receipt of $\mathrm{C} \& \mathrm{~S}$ reports were assessed.

\section{Retrospective data}

Data from C\&S reports were collected to identify a statistically significant reduction in sensitivity to antibiotics in the preceding 2 years (September 2016 to August 2017 and September 2017 to August 2018) among common uropathogens (Escherichia coli, Klebsiella isolates, and Pseudomonas aeruginosa). Between September 2016 and August 2017, 233 E. coli, 270 Klebsiella isolates, and 102 P. aeruginosa isolates and between September 2017 and August 2018, 132 E. coli, 169 Klebsiella isolates, and 59 P. aeruginosa isolates were identified with significant bacteriuria from HA-UTI data. Between September 2016 and August 2017, 413 E. coli, 209 Klebsiella isolates, and 43 P. aeruginosa isolates and between September 2017 and August 2018, 267 E. coli, 127 Klebsiella isolates, and $43 P$. aeruginosa isolates were identified with significant bacteriuria from CA-UTI data.

\section{Statistical analysis}

Data were compiled using Microsoft Excel and statistical analysis was conducted using IBM SPSS version 20. Descriptive statistical methods were used to calculate mean \pm standard deviation, median, and range for relevant parameters. Pearson's chi-square test was carried out in the retrospective study to determine whether the reduction in sensitivity to antibiotics observed between the preceding 2 years (September 2016-August 2017 and September 2017-August 2018) was statistically significant; $p$-values $<0.05$ was taken as significant (Figs. 2 and 3).

\section{RESULTS AND DISCUSSION}

\section{Prospective study}

\section{Demographics and risk factors}

The HA-UTI study population consisted of $57.7 \%$ males and $42.3 \%$ females, with a mean age of $61.96 \pm 15.51$ years (median age 65 years, range 18-91 years) and the CA- 

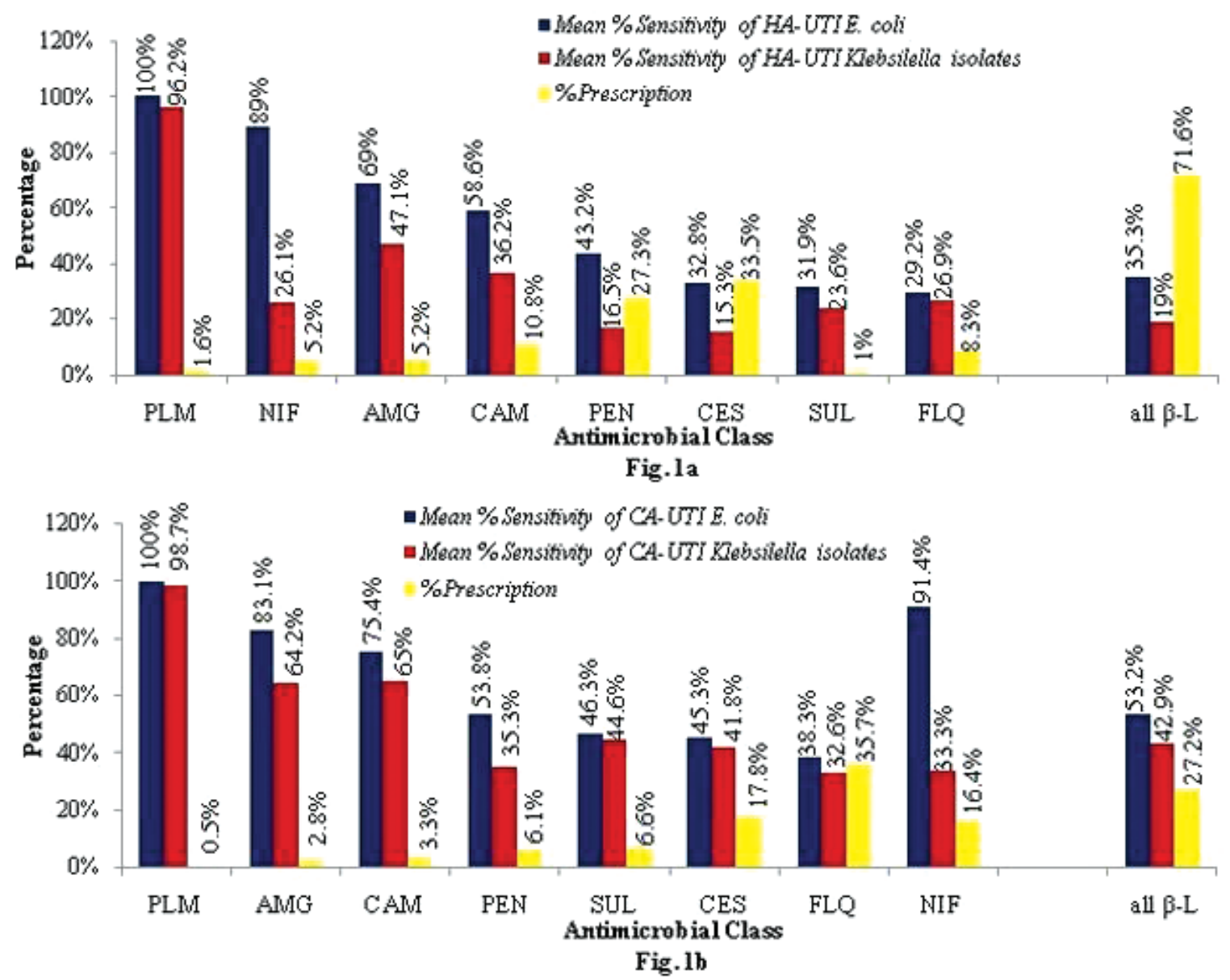

Figure 1. (a and b) Comparison of the mean percentage sensitivity (of most prevalent uropathogens in HA-UTI and CA-UTI, respectively) for an antimicrobial class with the percentage antibiotic prescription from that antimicrobial class. Increased prescription of antimicrobials was accompanied by decreased sensitivity to that antimicrobial class. PLM = polymixins; $\mathrm{NIF}=$ nitrofurans; $\mathrm{AMG}=$ aminoglycosides; $\mathrm{CAM}=$ carbapenems; $\mathrm{PEN}=$ penicillins; $\mathrm{CES}=$ cephalosporins; $\mathrm{SUL}=$ sulfonamides; $\mathrm{FLQ}=$ fluoroquinolones; all $\beta-\mathrm{L}=$ all $\beta$-Lactams.

UTI study population consisted of $53.4 \%$ females and $46.6 \%$ males, with a mean age of $55.46 \pm 16.34$ years (median age 57 years, range 18-92 years). Females have a higher incidence of uncomplicated CA-UTI due to shorter and wider urethra and its proximity to the anus. Elderly patients ( $>50$ years) are more likely to acquire UTI due to decreased immune function and increased number and duration of hospital admissions, similar to the study conducted by Karishetti and Shaik (2019). Females had a higher prevalence in the age groups $<40$ years in both HAUTI and CA-UTI and $>80$ years in HA-UTI population. This is due to increased sexual activity and occurrence of pregnancies in lower age groups. Higher risk in post-menopausal women is due to alterations in bladder emptying and changes in vaginal flora with loss of estrogen and Lactobacilli. Males $>40$ years have a higher prevalence commonly due to an enlarged prostate, resultant ailments, and urogenital instrumentation; similar results were observed by Shevade and Agarwal (2013). Predisposing factors observed in the HA-UTI and CA-UTI study populations were diabetes mellitus ( $48 \%$ and $54.3 \%$ ), catheterization $(51.1 \%$ and $12.8 \%)$, recurrent UTI $(17.8 \%$ and $23 \%$ ) benign prostatic hyperplasia $(10.6 \%$ and $13.1 \%)$, pregnancy $(0 \%$ and $4.5 \%)$, and other factors (such as urinary tract calculi and other kidney diseases, $45.3 \%$ and $27.5 \%$, respectively). Catheterization was the major risk factor of HAUTI in the study conducted by Melaku et al. (2012), where the risk of developing HA-UTI in catheterized patients was 2.6 times higher than non-catheterized patients. This can be due to increased use in inpatients, improper handling, and formation of biofilm. Diabetes mellitus often leads to UTI due to reduced immunity, diabetic nephropathy, peripheral neuropathy, and hyperglycemia that promote colonization in urinary tract. Diabetes mellitus was the major risk factor for CA-UTI, which is in accordance with Eshwarappa et al. (2011) and Chandrasekhar et al.'s (2018) studies.

\section{Prevalence}

Table 1 shows the prevalence of uropathogens in HAUTI and CA-UTI. Among the 370 HA-UTI isolates, Gram- 


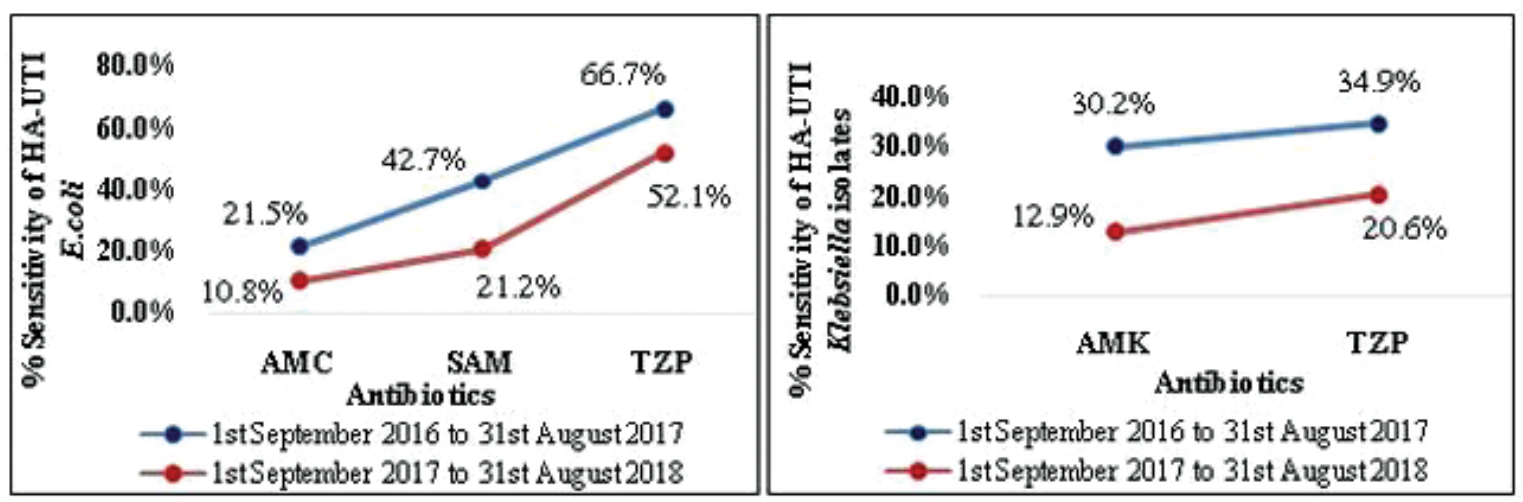

Figure 2. Statistically significant $(p<0.05)$ decrease in sensitivity of HA-UTI isolates between the preceding 2 years assessed using Pearson's chi-square test: $E$. coli to SAM $=$ ampicillin/sulbactam $(p=0.001) ;$ AMC $=$ amoxicillin $/$ clavulanate $(p=0.014)$; and TZP = piperacillin/tazobactam $(p=0.009)$, and Klebsiella isolates to AMK $=\operatorname{amikacin}(p=0.001)$ and TZP $=$ piperacillin/tazobactam $(p=0.007)$.

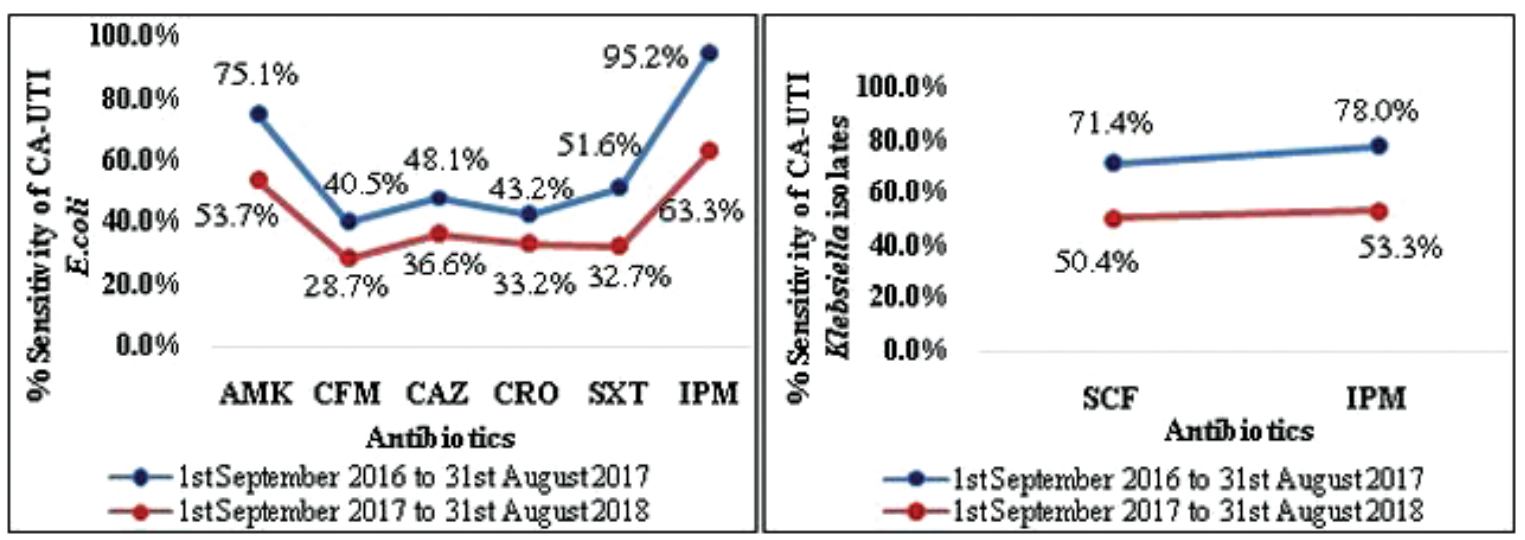

Figure 3. Statistically significant $(p<0.05)$ decrease in sensitivity of CA-UTI isolates between the preceding 2 years assessed using Pearson's chi-square test: $E$. coli to AMK = amikacin $(p=0.001) ; \mathrm{CFM}=$ cefixime $(p=0.002) ; \mathrm{CAZ}=$ ceftazidime $(p=0.040) ; \mathrm{CRO}=$ ceftriaxone $(p=0.009)$; SXT = cotrimoxazole $(p=0.022)$; and IPM = imipenem $(p=0.001)$, and Klebsiella isolates to SCF $=$ cefoperazone/ $\operatorname{sulbactam}(p=0.001)$ and IPM $=$ imipenem $(p=0.001)$.

negative bacilli (GNB) were the predominant cause of infection (68.6\%), followed by Gram-positive cocci (GPC) (16.5\%) and fungi $(14.9 \%)$. E. coli was the most prevalent HA-UTI pathogen (19.7\%), followed by Klebsiella pneumoniae (19.5\%) and P. aeruginosa $(14.3 \%)$. Klebsiella isolates (K. pneumoniae and other Klebsiella spp.) were seen in a total of $24.1 \%$ of isolates. The study conducted by Dharmishtha et al. (2012) and Kamat et al. (2009) also found $E$. coli to be the most prevalent HA-UTI uropathogen.

Among the 351 CA-UTI isolates, GNB was found in $89.7 \%$ isolates, followed by GPC $(9.1 \%)$ and fungi $(1.1 \%)$. E. coli was the most prevalent CA-UTI pathogen (53.6\%), followed by $K$. pneumoniae $(22.2 \%)$ and Streptococcus spp. (3.4\%). Klebsiella isolates (K. pneumoniae, Klebsiella oxytoca, and other Klebsiella spp.) were seen in a total of $25.9 \%$ of isolates. Similar findings by Dharmishtha et al. (2012) showed E. coli to be the most prevalent CA-UTI uropathogen. Polymicrobial infections were seen in $9.4 \%$ and $4.8 \%$ of study patients with HA-UTI and CA-UTI, respectively, and were commonly among catheterized patients as long-term catheterization often leads to polymicrobial infections (Kamat et al., 2009).
Multiple drug resistance and multiple antibiotic resistance index

MDR was identified in $47 \%$ and $46.2 \%$ of HA-UTI and CA-UTI isolates, respectively. Among the MDR isolates in each group, the highest was Klebsiella isolates $(41.95 \%)$ followed by E. coli (27\%) in HA-UTI and E. coli (52.5\%) followed by Klebsiella isolates $(35.8 \%)$ in CA-UTI. The alarmingly high number of MDR isolates is of major concern and is probably because majority of the patients are elderly and who are prone to repeated infections, leading to multiple exposures to antibiotics which indeed promotes resistance. The mean MARI for the MDR uropathogens was found to be $>0.5$ in HA-UTI and $\geq 0.2$ in CA-UTI. Thus, indicating that both hospital and community are "high-risk" sources of antibiotic resistance with frequent misuse or overuse of antibiotics. HA-UTI isolates have a higher mean MARI due to increased intensity of antimicrobial use in hospital and ineffective infection control, while in CAUTI it indicates probable misuse of antimicrobials by over the counter dispensing of antibiotics, OP prescriptions for nonbacterial infections, self-medication, poor adherence to dosage 
Table 1. Prevalence of uropathogens in HA-UTI and CA-UTI study participants.

\begin{tabular}{|c|c|c|c|c|c|}
\hline & \multirow{2}{*}{ Uropathogens } & \multicolumn{2}{|c|}{ HA-UTI } & \multicolumn{2}{|c|}{ CA-UTI } \\
\hline & & No. & $\%$ & No. & $\%$ \\
\hline \multirow{20}{*}{$\overbrace{\circlearrowleft}^{\oplus}$} & Non-fermenter Acinetobacter spp. & 9 & 2.4 & 3 & 0.85 \\
\hline & Burkholderia cepacia & 1 & 0.27 & - & - \\
\hline & C. koseri & 2 & 0.5 & 5 & 1.4 \\
\hline & Citrobacter freundii & 1 & 0.27 & 1 & 0.28 \\
\hline & Chryseobacterium indologenes & 4 & 1.1 & - & - \\
\hline & E. coli & 73 & 19.7 & 188 & 53.6 \\
\hline & Enterobacter spp. & 4 & 1.1 & 2 & 0.6 \\
\hline & Enterobactercloacae complex & - & - & 2 & 0.6 \\
\hline & K. pneumoniae & 72 & 19.5 & 78 & 22.2 \\
\hline & K. oxytoca & - & - & 2 & 0.6 \\
\hline & Klebsiella spp. & 17 & 4.6 & 11 & 3.1 \\
\hline & Morganella morganii & 2 & 0.5 & - & - \\
\hline & Myroides spp & 1 & 0.27 & - & - \\
\hline & Proteus mirabilis & 3 & 0.8 & 4 & 1.1 \\
\hline & Proteus vulgaris & - & - & 1 & 0.28 \\
\hline & Providencia rettgeri & 8 & 2.2 & 3 & 0.85 \\
\hline & P. aeruginosa & 53 & 14.3 & 11 & 3.1 \\
\hline & Serratia marcescens & 4 & 1.1 & 4 & 1.1 \\
\hline & E. faecalis & 14 & 3.8 & 6 & 1.7 \\
\hline & Enterococcus spp. & 33 & 8.9 & 5 & 1.4 \\
\hline \multirow{6}{*}{ 仓े } & E. faecium & 10 & 2.7 & - & - \\
\hline & Staphylococcus aureus & 2 & 0.5 & 7 & 2 \\
\hline & Staphylococcus saprophyticus & - & - & 2 & 0.6 \\
\hline & Streptococcus spp. & 2 & 0.5 & 12 & 3.4 \\
\hline & Candida glabrata & - & - & 1 & 0.28 \\
\hline & Candida albicans & 14 & 3.8 & - & - \\
\hline \multirow{5}{*}{ ত্ } & C. non-albicans & 21 & 5.7 & 1 & 0.28 \\
\hline & Candida guillermondii & 1 & 0.27 & - & - \\
\hline & Candida spp. & 1 & 0.27 & - & - \\
\hline & Candida tropicalis & 18 & 4.9 & 2 & 0.6 \\
\hline & TOTAL & 370 & 100 & 351 & 100 \\
\hline
\end{tabular}

regimens, antibiotic pollution, and overuse of antibiotics for other purposes, like in growth promoters for livestock. Hence, it is important to promote awareness regarding the rational use of antibiotics to the physicians, pharmacists, and patients. In the study conducted by Prakash and Saxena (2013), the MARI for HA-UTI ranged from 0.33 to 0.83 and for CA-UTI it ranged from 0.16 to 0.77 . Table 2 gives the MDR, mean MARI, and MARI range of HA-UTI and CA-UTI study isolates.

\section{Antimicrobial susceptibility of uropathogens and prescribing pattern of antimicrobials}

Tables 3 and 4 represent the antibiograms of HAUTI and CA-UTI isolates. In HA-UTI, E. coli had the highest sensitivity to drugs like colistin (CST), amikacin, meropenem (MEM), cefoperazone/sulbactam (SCF), and piperacillin/tazobactam (TZP). Klebsiella isolates had the highest sensitivity to CST and amikacin. MEM, SCF, TZP, and nirofurantoin showed $<40 \%$ sensitivity to Klebsiella isolates. Pseudomonas aeruginosa showed 100\% sensitivity to CST and $<35 \%$ sensitivity to all other drugs. All GNB showed $<30 \%-$ $40 \%$ sensitivity toward common antibiotics like cefixime, ceftriaxone (CRO), amoxicillin/clavulanate, ciprofloxacin (CIP), and cotrimoxazole. Similar sensitivities were shown by E. coli in the study by Kamat et al. (2009). GPC showed a higher sensitivity to linezolid and vancomycin, while sensitivity to CIP was low. Enterococcus spp. and Enterococcus faecium had low sensitivity for ampicillin/amoxicillin and nitrofurantoin (NIT), while Enterococcus faecalis showed $>60 \%$ for these drugs. Fungal HA-UTI isolates had $100 \%$ sensitivity to amphotericin $\mathrm{B}$ and fluconazole.

In CA-UTI, E. coli had the highest sensitivity to CST, MEM, amikacin, NIT, TZP, and SCF. Chandrasekhar et al. (2018) and Bardoloi and Babu (2017) suggested NIT as drug of choice for empirical treatment due to its higher sensitivity. Klebsiella isolates had the highest sensitivity to CST, MEM, and amikacin, whereas NIT sensitivity was only $33.3 \%$. $P$. aeruginosa showed $100 \%$ sensitivity to CST and 50\% sensitivity to TZP, while all other drugs had $<45 \%$ sensitivity. Common antibiotics like cefixime, amoxicillin/clavulanate, CRO, CIP, and cotrimoxazole had $<50 \%$ sensitivity to E. coli and Klebsiella isolates. Streptococcus spp. had high susceptibility to CRO, ofloxacin, vancomycin, linezolid, and ampicillin/amoxicillin. Only four fungal CA-UTI isolates were present, hence we did not include them in the antibiogram. The HA-UTI isolates had higher resistance than CA-UTI isolates.

A total of $57.7 \%$ of HA-UTI patients received empirical therapy, out of which $52.9 \%$ of the patients were resistant to the antimicrobial. After the C\&S results, $77.6 \%$ of the participants received antimicrobials, out of which $34.6 \%$ of the patients received resistant empirical therapy and required a change in antibiotics. The most common empirical antibiotic was TZP, while the most common antibiotics prescribed after the C\&S results were MEM and TZP. A common class of empirical antibiotics prescribed was cephalosporins (33.5\%), followed by penicillins $(27.3 \%)$ and carbapenems $(10.8 \%)$. A common class of antibiotics used after the C\&S results were penicillins $(20.2 \%)$, followed by cephalosporins $(19.4 \%)$ and carbapenems $(16 \%)$. The change in percentage of common antibiotic class before and after the C\&S results was because of high MDR isolates.

A total of $57.3 \%$ of CA-UTI patients received empirical antibiotics, out of which $47.9 \%$ of the patients were resistant to the agent due to increased use of ß-lactams and fluoroquinolones with lower sensitivity. The commonly used empirical agent was NIT $(16.4 \%)$, followed by levofloxacin (LVX) $(14.6 \%)$ and cefixime $(10.8 \%)$. Fluoroquinolones $(35.7 \%)$ were the most commonly prescribed class of antimicrobials in empirical treatment of CAUTI, followed by cephalosporins (17.8\%) and nitrofurans (16.4\%), which is similar to the study by Prakasam et al. (2012). After the C\&S results, $60.6 \%$ of the study patients received antimicrobials, out of which $31.5 \%$ of the patients received resistant empirical 
Table 2.MDR, mean MARI, and MARI range of HA-UTI and CA-UTI study isolates

\begin{tabular}{|c|c|c|c|c|c|c|c|c|}
\hline \multirow{3}{*}{ Uropathogens } & \multicolumn{4}{|c|}{ HA-UTI } & \multicolumn{4}{|c|}{ CA-UTI } \\
\hline & \multicolumn{2}{|c|}{$\begin{array}{c}\text { MDR Isolates ( } n \\
=174)\end{array}$} & \multirow{2}{*}{$\begin{array}{l}\text { Mean } \\
\text { MARI }\end{array}$} & \multirow{2}{*}{ MARI range } & \multicolumn{2}{|c|}{$\begin{array}{l}\text { MDR Isolates ( } n \\
=162)\end{array}$} & \multirow{2}{*}{$\begin{array}{l}\text { Mean } \\
\text { MARI }\end{array}$} & \multirow[t]{2}{*}{ MARI range } \\
\hline & No. & $\%$ & & & No. & $\%$ & & \\
\hline Citrobacter koseri & 1 & 0.57 & 0.72 & - & - & - & - & - \\
\hline C. freundii & - & - & - & - & 1 & 0.62 & 0.91 & - \\
\hline Enterobacter spp. & 3 & 1.72 & 0.73 & $0.57-0.81$ & 2 & 1.23 & 0.23 & $0.2-0.26$ \\
\hline Enterococcus spp. & 3 & 1.72 & 0.69 & $0.23-0.94$ & 1 & 0.62 & 0.8 & - \\
\hline E. coli & 47 & 27 & 0.63 & $0.18-0.93$ & 85 & 52.5 & 0.57 & $0.23-0.94$ \\
\hline Klebsiella isolates & 73 & 41.95 & 0.86 & $0.49-1.0$ & 58 & 35.8 & 0.73 & $0.11-0.94$ \\
\hline M. morganii spp. & 1 & 0.57 & 0.58 & - & - & - & - & - \\
\hline Myroides spp. & 1 & 0.57 & 1 & - & - & - & - & - \\
\hline Non fermenter Acinetobacter spp. & 5 & 2.9 & 0.79 & $0.52-0.93$ & 1 & 0.62 & 0.86 & - \\
\hline Proteus spp. & 1 & 0.57 & 0.68 & - & 2 & 1.23 & 0.4 & $0.37-0.43$ \\
\hline P. rettgeri & 9 & 5.2 & 1 & - & 2 & 1.23 & 0.83 & $0.7-0.95$ \\
\hline P. aeruginosa & 28 & 16.1 & 0.92 & $0.92-0.93$ & 8 & 4.9 & 0.83 & $0.35-0.93$ \\
\hline S. marcescens & 2 & 1.15 & 0.83 & $0.78-0.89$ & 1 & 0.62 & 0.28 & - \\
\hline S. aureus & - & - & - & - & 1 & 0.62 & 0.38 & - \\
\hline
\end{tabular}

Table 3. Percentage susceptibility (no. of isolates susceptible/total no. of isolates tested) of GNB from HA-UTI and CA-UTI isolates.

\begin{tabular}{|c|c|c|c|c|c|c|}
\hline \multirow{3}{*}{$\begin{array}{c}\text { Uropathogens } \\
\text { Total Isolates }\end{array}$} & \multicolumn{2}{|c|}{ E. coli } & \multicolumn{2}{|c|}{ Klebsiella isolates ${ }^{\mathrm{a}}$} & \multicolumn{2}{|c|}{ P. aeruginosa } \\
\hline & \multirow{2}{*}{$\frac{73}{\text { HA-UTI }}$} & \multirow{2}{*}{$\frac{188}{\text { CA-UTI }}$} & \multirow{2}{*}{$\frac{89}{\text { HA-UTI }}$} & \multirow{2}{*}{$\frac{91}{\text { CA-UTI }}$} & \multirow{2}{*}{$\frac{53}{\text { HA-UTI }}$} & \multirow{2}{*}{$\frac{11}{\text { CA-UTI }}$} \\
\hline & & & & & & \\
\hline \multicolumn{7}{|c|}{ Percentage susceptibility (no. of isolates susceptible / Total no. of isolates tested) } \\
\hline AMK & $79.2(57 / 72)$ & $92.1(175 / 190)$ & $51.7(46 / 89)$ & $67.4(62 / 92)$ & $30.2(16 / 53)$ & $36.4(4 / 11)$ \\
\hline SAM & $62.5(45 / 72)$ & $72.5(137 / 189)$ & $25.8(23 / 89)$ & $45.1(41 / 91)$ & $0(0 / 2)$ & - \\
\hline AMC & $34.4(21 / 61)$ & $32.9(48 / 146)$ & $24.3(18 / 74)$ & $30.1(22 / 73)$ & $0(0 / 1)$ & - \\
\hline FEP & $16.7(2 / 12)$ & $41.4(12 / 29)$ & $8(2 / 25)$ & $31.3(5 / 16)$ & $28(14 / 50)$ & $40(4 / 10)$ \\
\hline CFM & $23.6(17 / 72)$ & $30(57 / 190)$ & $12.2(11 / 90)$ & $38.5(35 / 91)$ & - & - \\
\hline CFP & $29.2(21 / 72)$ & $37.8(70 / 185)$ & $13.6(12 / 88)$ & $40.7(37 / 91)$ & $29.2(7 / 24)$ & $40(2 / 5)$ \\
\hline $\mathrm{SCF}$ & $66.2(47 / 71)$ & $87.9(160 / 182)$ & $26.9(24 / 89)$ & $55.4(51 / 92)$ & $30.8(16 / 52)$ & $18.18(2 / 11)$ \\
\hline CAZ & $33.3(8 / 24)$ & $37.1(33 / 89)$ & $13.3(4 / 30)$ & $44.4(20 / 45)$ & $30.8(16 / 52)$ & $20(2 / 10)$ \\
\hline $\mathrm{CRO}$ & $27.9(19 / 68)$ & $37.8(65 / 172)$ & $17.6(15 / 85)$ & $40.5(34 / 84)$ & - & - \\
\hline CIP & $29.2(21 / 72)$ & $38.3(72 / 188)$ & $26.9(24 / 89)$ & $32.6(30 / 92)$ & $29.4(15 / 51)$ & $9.1(1 / 11)$ \\
\hline CST & $100(53 / 53)$ & $100(134 / 134)$ & $96.2(76 / 79)$ & $98.7(77 / 78)$ & $100(49 / 49)$ & $100(10 / 10)$ \\
\hline SXT & $31.9(23 / 72)$ & $46.3(88 / 190)$ & $23.6(21 / 89)$ & $44.6(41 / 92)$ & $0(0 / 1)$ & - \\
\hline GEN & $58.8(40 / 68)$ & $74.1(137 / 185)$ & $42.5(37 / 87)$ & $60.9(56 / 92)$ & $30.6(15 / 49)$ & $27.3(3 / 11)$ \\
\hline IPM & $41.7(25 / 60)$ & $57.1(76 / 133)$ & $33.8(25 / 74)$ & $54.8(40 / 73)$ & $17.1(7 / 41)$ & $10(1 / 10)$ \\
\hline LVX & $50(1 / 2)$ & - & $0(0 / 1)$ & $0(0 / 1)$ & $28.8(15 / 52)$ & $9.1(1 / 11)$ \\
\hline MEM & $75.4(49 / 65)$ & $93.6(161 / 172)$ & $38.6(32 / 83)$ & $75.3(64 / 85)$ & $28(14 / 50)$ & $36.4(4 / 11)$ \\
\hline NIT & $89(65 / 73)$ & $91.4(171 / 187)$ & $26.1(23 / 88)$ & $33.3(30 / 90)$ & $0(0 / 1)$ & - \\
\hline PIP & $14.3(3 / 21)$ & $22.4(15 / 67)$ & $4.3(1 / 23)$ & $25.8(8 / 31)$ & $30.8(16 / 52)$ & $36.4(4 / 11)$ \\
\hline $\mathrm{TZP}$ & $66.2(43 / 65)$ & $89.1(163 / 183)$ & $24.4(20 / 82)$ & $51.2(44 / 86)$ & $34.7(17 / 49)$ & $50(5 / 10)$ \\
\hline TIM & $38.6(17 / 44)$ & $52(52 / 100)$ & $3.8(2 / 53)$ & $24.5(12 / 49)$ & $26.7(8 / 30)$ & $0(0 / 4)$ \\
\hline TOB & - & - & - & $100(1 / 1)$ & $30.8(16 / 52)$ & $27.3(3 / 11)$ \\
\hline
\end{tabular}

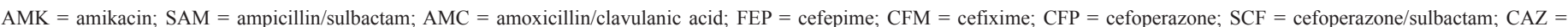

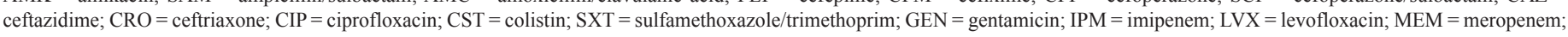
$\mathrm{NIT}=$ nitrofurantoin; $\mathrm{PIP}=$ piperacillin; $\mathrm{TZP}=$ piperacillin/tazobactam; $\mathrm{TIM}=$ ticarcillin $/$ clavulanic acid $; \mathrm{TOB}=$ tobramycin.

${ }^{a}$ Klebsiella isolates includes K. pneumoniae and other Klebsiella spp.

${ }^{\mathrm{b}}$ Antimicrobial agents. 
Table 4. Percentage susceptibility (no. of isolates susceptible/total no. of isolates tested) of GPC and fungi from HA-UTI and CA-UTI isolates.

\begin{tabular}{|c|c|c|c|c|c|c|c|c|}
\hline \multicolumn{2}{|r|}{ Uropathogens } & Strepto-coccus spp. & Entero-coccus spp. & E. faecium & E. faecalis & C. non-albicans & C. albicans & C. tropicalis \\
\hline \multirow{2}{*}{\multicolumn{2}{|c|}{ Total Isolates }} & 12 & 33 & 10 & 14 & 21 & 14 & 18 \\
\hline & & CA-UTI & HA-UTI & HA-UTI & HA-UTI & HA-UTI & HA-UTI & HA-UTI \\
\hline & & \multicolumn{7}{|c|}{ Percentage susceptibility (No. of isolates susceptible/total no. of isolates tested) } \\
\hline \multirow{16}{*}{ 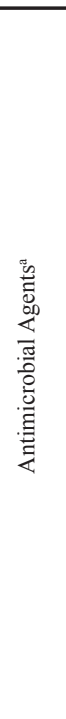 } & AMP-AMX & $100(9 / 9)$ & $3.2(1 / 31)$ & $0(0 / 9)$ & $69.2(9 / 13)$ & - & - & - \\
\hline & CRO & $91.7(11 / 12)$ & - & - & - & - & - & - \\
\hline & CIP & $100(1 / 1)$ & $0(0 / 31)$ & $0(0 / 10)$ & $21.4(3 / 14)$ & - & - & - \\
\hline & CLI & $100(6 / 6)$ & $87.5(14 / 16)$ & $50(1 / 2)$ & $85.7(6 / 7)$ & - & - & - \\
\hline & LVX & - & $87.5(14 / 16)$ & $20(1 / 5)$ & $85.7(6 / 7)$ & - & - & - \\
\hline & LZD & $100(7 / 7)$ & $100(31 / 31)$ & $90(9 / 10)$ & $100(10 / 10)$ & - & - & - \\
\hline & NIT & $100(6 / 6)$ & $14.3(5 / 35)$ & $10(1 / 10)$ & $84.6(11 / 13)$ & - & - & - \\
\hline & OFX & $83.3(10 / 12)$ & - & - & - & - & - & - \\
\hline & PENG & $90.9(10 / 11)$ & $2.9(1 / 34)$ & $0(0 / 9)$ & $61.5(8 / 13)$ & - & - & - \\
\hline & TEC & $100(7 / 7)$ & $86.9(20 / 23)$ & $50(3 / 6)$ & $100(8 / 8)$ & - & - & - \\
\hline & TET & - & $34.4(11 / 32)$ & $20(2 / 10)$ & $30.8(4 / 13)$ & - & - & - \\
\hline & VAN & $100(7 / 7)$ & $90.3(28 / 31)$ & $70(7 / 10)$ & $100(9 / 9)$ & - & - & - \\
\hline & AMB & - & - & - & - & 100 & 100 & 100 \\
\hline & & & & & & $(21 / 21)$ & $(14 / 14)$ & $(18 / 18)$ \\
\hline & FLC & - & - & - & - & 100 & 100 & \\
\hline & & & & & & $(21 / 21)$ & $(14 / 14)$ & $100(18 / 18)$ \\
\hline
\end{tabular}

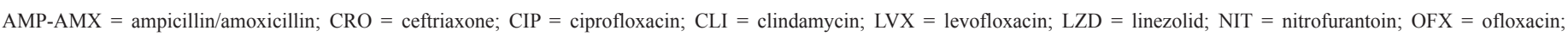
$\mathrm{PENG}=$ penicillin $\mathrm{G} ; \mathrm{TEC}=$ teicoplanin; $\mathrm{TET}=$ tetracycline; $\mathrm{VAN}=$ vancomycin; $\mathrm{AMB}=$ amphotericin $\mathrm{B} ; \mathrm{FLC}=$ fluconazole.

aAntimicrobial agents.

therapy and required a change in antibiotics. Nitrofurans $(25.5 \%)$ were the most prescribed class of antimicrobials after the C\&S results, followed by fluoroquinolones (19.3\%) and cephalosporins $(16.9 \%)$.

Figure 1a and $\mathrm{b}$ shows the comparison of mean percentage sensitivity of most prevalent uropathogens with the percentage antibiotic prescription of an antimicrobial class. In both HA-UTI and CA-UTI, increased prescription of antimicrobials was accompanied by decreased sensitivity to that antimicrobial class. This is because increased use causes antibiotics to exert selective pressure which eventually leads to resistance. Low sensitivity leads to treatment failure, resulting in repeated exposures to multiple antibiotics, which further accelerates resistance. HA-UTI isolates had higher sensitive to reserve drugs like CST, vancomycin, and linezolid that are not frequently used in the hospital, while common agents, third generation cephalosporins and penicillins, had marked decrease in sensitivity. Carbapenem use for HA-UTI had increased due to an increase in MDR and hence reflected in the sensitivity to the drug, which has surprisingly decreased in Klebsiella isolates and $P$. aeruginosa. As seen in the graph, $71.6 \%$ of empirically prescribed antibiotics were $\beta$-lactams and hence had significant reduction in sensitivity for HA-UTI isolates. Oral agents, like CIP (fluoroquinolones), several cephalosporins, and cotrimoxazole, whose increasing resistance have been of concern for years worldwide had a marked decrease in sensitivity with only NIT maintaining higher sensitivity in both HA-UTI and CA-UTI. This has detrimental consequences on prophylaxis for infections, like those after urological interventions, which place at risk the ability to treat common infections in community and hospital (Munoz-Davila, 2014). ß-lactams like cefixime, LVX, and cotrimoxazole are widely prescribed for CA-UTI, while the global resistance rate of all uropathogens to CIP, cephalosporins, and penicillins is more than $50 \%, 35 \%-50 \%$, and $50 \%$, respectively (Cek et al., 2014), thereby increasing the potential for resistance. NIT, despite being commonly prescribed in CA-UTI, has high sensitivity, probably due to its multiple mechanisms of action. A clinically significant correlation between antibiotic usage and resistance pattern was shown in the study conducted by Kamat et al. (2008), which is similar to our study where antibiotics frequently prescribed had a higher resistance than those prescribed less frequently.

\section{Retrospective study}

Antibiotic susceptibility trends in HA-UTI isolates (Fig. 2) in the preceding 2 years showed a statistically significant decrease in sensitivity of (a) E. coli to ampicillin/sulbactam $(p=0.001)$, amoxicillin/clavulanate $(p=0.014)$, and TZP $(p=$ $0.009)$; and (b) Klebsiella isolates to amikacin $(p=0.001)$ and TZP $(p=0.007)$. $P$. aeruginosa sensitivity for antibiotics showed no statistically significant difference. The percentage of MDR isolates in both years was $>50 \%$ of total isolates for $E$. coli and P. aeruginosa, while it was $>75 \%$ of total isolates for Klebsiella isolates.

Figure 3 CA-UTI isolates showed a statistically significant decrease in sensitivity of (a) E. coli to amikacin ( $p=$ 
$0.001)$, cefixime $(p=0.002)$, ceftazidime (CAZ) $(p=0.040)$, CRO $(p=0.009)$, cotrimoxazole $(p=0.022)$, and imipenem (IPM) ( $p$ $=0.001)$; and (b) Klebsiella isolates to SCF $(p=0.001)$ and IPM $(p=0.001)$. P. aeruginosa sensitivity for antibiotics showed no statistically significant difference. The percentage of MDR isolates increased from $36.1 \%$ in the first year to $41.9 \%$ in the second year for E. coli, from $49.3 \%$ to $49.6 \%$ for Klebsiella isolates, and from $34.9 \%$ to $53.5 \%$ for $P$. aeruginosa.

Asia is known as the resistance epicenter due to its high prevalence rate of extended spectrum b-lactamase enzymes amongst E. coli and Klebsiella isolates, compared to North America and Europe (Jean et al., 2016). A statistically significant reduction in sensitivity of antibiotics in our study is indicative of the increasing number of MDR strains and Extended Spectrum Beta-Lactamase (ESBL) producers among these isolates mainly due to overuse of these antibiotics. The emergence of resistance in UTI is of concern since majority of uropathogens are GNB (Mitchell et al., 2016). Antimicrobial resistance, particularly the most common being $E$. coli, is directly associated with prescribing in primary care (Bhat and Sarkar, 2011). Resistance limits the options for treatment of various infections due to the decrease in development of new antibiotics.

Strengthening of antimicrobial stewardship strategies is the need of the hour. Empirical antimicrobial policies must be developed and regularly updated by continuous antimicrobial surveillance on prevalence and susceptibility of uropathogens since it varies with time and locality. Appropriate use of antimicrobials must be promoted through post-prescription reviews and feedback, antimicrobial utilization review programs, and auditing of dispensing practices. Monitoring antimicrobial usage, including indications, quantity, and pattern of use with restriction on use of carbapenems, fluoroquinolones, and third-generation cephalosporins is important. De-escalation from broad-spectrum antibiotics to narrower-spectrum antibiotics once the susceptibility data are available is essential. Reintroduction of old antimicrobials, like fosfomycin, is seen which is a potential old drug now used for the treatment of drug-resistant UTI and studies has shown promising results (Patwardhan and Singh, 2017; Prakash et al., 2009; Pullukcu et al., 2007). Effective infection control strategies and educating prescribers, dispensers, patients, and the general community on the appropriate use of antimicrobials is necessary.

Such measures will avoid overuse of broad-spectrum antibiotics and monitors resistance trends within an institution, thus optimizing treatment and improving rational prescription and use of antimicrobials. The overall goal should be to reduce the total consumption of antimicrobials and alter its usage in favor of regimens less likely to promote the emergence of resistant strains.

\section{CONCLUSION}

E. coli was the most prevalent uropathogen in both HAUTI and CA-UTI. Study patients in both groups showed alarmingly high MDR isolates with mean MARI value of $>0.2$. Percentage susceptibility of common uropathogens to frequently prescribed antimicrobials was low. Statistically significant reduction in antimicrobial sensitivity to commonly prescribed agents in the past years indicates irrational use of antimicrobials. The need of the hour is to strengthen antimicrobial stewardship strategies, conduct continuous institutional surveillance on prevalence and susceptibility of uropathogens, and promote rational prescription and use of antimicrobials.

\section{AUTHORS' CONTRIBUTIONS}

Achukutty Tomy, Aravind H, Emil David, and Ravina Ravi: conceptualization, investigation, formal analysis, visualization, and writing (original draft). Lakshmi R and Appu Thomas: supervision and writing (review and editing).

\section{CONFLICT OF INTEREST}

All authors declare that they have no conflict of interest.

\section{FUNDING}

None.

\section{REFERENCES}

Agaba P, Tumukunde J. Tindimwebwa JV, Kwizera A. Nosocomial bacterial infections and their antimicrobial susceptibility patterns among patients in Ugandan intensive care units: a cross sectional study. BMC Res Notes, 2017; 10:349-61.

Aguilar-Duran S, Horcajada JP, Sorlỉ L, Montero M, Salvadò M, Grau S, Gòmez J, Knobel H. Community-onset healthcare-related urinary tract infecions: comparison with community and hospital-acquired urinary tract infection. J Infect, 2012; 64:478-83.

Bardoloi V, Babu KY. Comparative study of isolates from community-acquired and catheter-associated urinary tract infections with reference to biofilm-producing property, antibiotic sensitivity and multidrug resistance. J Med Microbiol, 2017; 66:927-36.

Bhat PV, Sarkar A. Emergence and control of multidrug resistant organisms in small cities in India: a wakeup call. Indian J Med Sci, 2011; $1: 337-43$.

Cek M, Tandoğdu Z, Wagelehner F, Tenke P, Naber K, BjerklundJohansen TE. Healthcare-associated urinary tract infections in hospitalized urological patients- a global perspective: results from the GPIU studies 2003-2010. World J Urol, 2014; 32:1587-94.

Chacko B, Thomas K, David T, Paul H, Jeyaseelan L, Peter JV. Attributable cost of a nosocomial infection in the intensive care unit: a prospective cohort study. World J Crit Care Med, 2017; 6:79-84.

Chandrasekhar D, Dollychan A, Roy BM, Cholamughath S, Parambil JC. Prevalence and antibiotic utilization pattern of uropathogens causing community-acquired urinary tract infection in Kerala, India. J Basic Clin Physiol Pharmacol, 2018; 29:671-7.

Dharmishtha TG, Paragi GJ, Kiran PN. A study on antibiotic related resistance in UTI patients: a comparison between community acquired and hospital acquired E. coli. Natl J Community Med, 2012; $3: 255-8$.

Eshwarappa M, Dosegowda R, Aprameya IV, Khan MW, Kumar PS, Kempegowda P. Clinico-microbiological profile of urinary tract infection in south India. Indian J Nephrol, 2011; 21:30-6.

Frimodt M. Correlation between pharmacokinetic/ pharmacodynamic parameters and efficacy for antibiotics in the treatment of urinary tract infection. Int J Antimicrob Agents, 2002; 19:546-53.

Hooton TM. The epidemiology of urinary tract infection and the concept of significant bacteriuria. Infection, 1990; 18:40-3.

Hooton TM, Bradley SF, Cardenas DD, Colgan R, Geerlings SE, Rice JC, Saint S, Schaeffer AJ, Tambayh PA, Tenke P, Nicolle LE. Diagnosis, prevention and treatment of catheter-associated urinary tract infection in adults: 2009 International Clinical Practice Guidelines from the Infectious Disease Society of America. Clin Infect Dis, 2010 $50: 625-63$. 
Jean SS, Coombs G, Ling T, Balaji V, Rodrigues C, Mikamo H, Kim MJ, Rajasekaram DG, Mendoza M, Tan TY, Kiratisin P, Ni Y, Weinman B, Xu Y, Hsueh PR. Epidemiology and antimicrobial susceptibility profiles of pathogens causing urinary tract infections in the Asia-Pacific region: results from the Study for monitoring antimicrobial resistance trends (SMART), 2010-2013. J Antimicrob Agents, 2016; 47:328-34.

Joseph AA, Odimayo MS, Olokoba LB, Olokoba AB, Popoola GO. Multiple antibiotic resistance index of Escherichia coli in a tertiary hospital South-West Nigeria. Med J Zambia, 2017; 44:225-32.

Kamat US, Fereirra A, Amonkar D, Motghare DD, Kulkarni MS. Epidemiology of hospital acquired urinary tract infections in a medical college hospital in Goa. Indian J Urol, 2009; 25:76-80.

Kamat US, Ferreira AM, Savio R, Motghare DD. Antimicrobial resistance among nosocomial isolates in a teaching hospital in Goa. Indian J Community Med, 2008; 33:89-92.

Karishetti MS, Shaik HB. Clinicomicrobial assessment of urinary tract infections in a tertiary care hospital. Indian J Health Sci Biomed Res, 2019; 12:69-74.

Kaur I, Grover IS, Singh J, Upveja KH, Paul S. Analysis of microbial resistance and preferances using antibiograms. J Infect Dis Ther, 2016; 4:302-7.

Köves B, Magyar A, Tenke P. Spectrum and antibiotic resistance of catheter-associate urinary tract infection. GMS Infect Dis, 2017; 5:1-5.

Magiorakos AP, Srinivasan A, Carey RB, Carmeli Y, Falagas ME, Giske CG, Harbarth S, Hindler JF, Kahlmeter G, Olsson-Liljequist B, Paterson DL, Rice LB, Stelling J, Struelens MJ, Vatopoulos A, Weber JT, Monnet DL. Multi-drug resistant, extensively drug-resistant and pandrugresistant bacteria: an international expert proposal for interim standard definitions for acquired resistance. Clin Microbiol Infect, 2012; 18:268-81.

Melaku S, Kibret M, Abera B, Gebre-Sellassie S. Antibiogram of nosocomial urinary tract infection in FelegeHiwot referral hospital, Ethiopia. Afr J Sci, 2012; 12:134-9.

Mitchell BG, Ferguson JK, Anderson M, Sear J, Barnett A. Length of stay and mortality associated with healthcare-associated urinary tract infection: a multi-state model. J Hosp Infect, 2016; 93:92-9.

Mukherjee M, Basu S, Mukherjee SK, Majumder M. Multidrug-resistance and extended spectrum beta-lactamase production in uropathogenic $E$. coli which were isolated from hospitalized patients in Kolkata, India. J Clin Diagn Res, 2013; 7:449-53.
Munoz-Davila MJ. Role of old antibiotics in the era of antibiotic resistance. Highlighted nitrofurantoin for the treatment of lower urinary tract infections. Antibiotics, 2014; 3:39-48.

Patwardhan V, Singh S. Fosfomycin for the treatment of drugresistant urinary tract infections: potential of an old drug not explored fully. Int Urol Nephrol, 2017; 49:1637-43.

Prakasam AK, Kumar KD, Vijayan M. A cross sectional study on distribution of urinary tract infection and their antibiotic utilisation pattern in Kerala. Int J Pharm Tech Res, 2012; 3:1125-30.

Prakash D, Saxena RS. Prevalence and antimicrobial susceptibility pattern of Escherichia coli in hospital and community acquired patients related to urinary tract infection in India. J App Pharm Sci, 2013; 3:124-32.

Prakash V, Lewis JS, Herrera ML, Wickes BL, Jorgenson JH. Oral and parentral therapeutic options for outpatient urinary infections caused by Enterobacteriaceae producing CTX-M extended-spectrum $\beta$-lactamases. Antimicrob Agents Chemother, 2009; 53:1278-80.

Pullukcu H, Tasbakan M, Sipahi OR, Yamazhan T, Aydemir S, Ulusoy S. Fosfomycin in the treatment of extended spectrum betalactamase-producing Escherichia coli-related lower urinary tract infections. Int J Antimicrob Agents, 2007; 29:62-5.

Shevade SU, Agarwal GN. Study of community and nosocomial uropathogens and their drug resistance. Natl J Community Med, 2013; $4: 647-52$

How to cite this article:

Tomy A, Hareendran A, David E, Ravi R, Ramachandran L, Thomas A. Antimicrobial susceptibility of uropathogens and prescribing patterns in hospital- and community-acquired urinary tract infections in a tertiary care hospital. J Appl Pharm Sci, 2020; 10(11):050-058. 